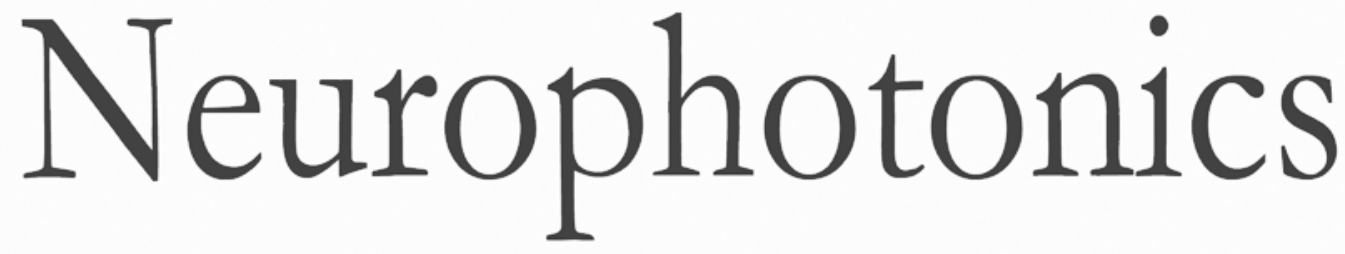

\title{
Bimorph deformable mirror-based adaptive optics scanning laser ophthalmoscope for the clinical design and performance
}

\author{
Yuanyuan Wang \\ Yi He \\ Ling Wei \\ Jinsheng Yang \\ Xiqi Li \\ Hong Zhou \\ Guohua Shi \\ Yudong Zhang
}




\title{
Bimorph deformable mirror-based adaptive optics scanning laser ophthalmoscope for the clinical design and performance
}

\author{
Yuanyuan Wang, ${ }^{a, b, c, \dagger}$ Yi He, ${ }^{c, d, e, *, \dagger}$ Ling Wei, ${ }^{c}$ Jinsheng Yang, ${ }^{c}$ Xiqi Li, ${ }^{c}$ Hong Zhou, ${ }^{c}$ Guohua Shi, ${ }^{d, e, \star}$ and \\ Yudong Zhang ${ }^{\mathrm{c}}$

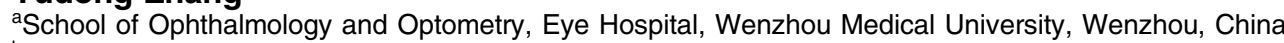 \\ bWenzhou Medical University, Wenzhou, China \\ ${ }^{\circ}$ Chinese Academy of Sciences, Key Laboratory on Adaptive Optics, Chengdu, China \\ dJiangsu Key Laboratory of Medical Optics, Suzhou, China \\ ${ }^{e}$ Chinese Academy of Sciences, Suzhou Institute of Biomedical Engineering and Technology, Suzhou, China
}

\begin{abstract}
We developed a clinical ophthalmic prototype by combining bimorph deformable mirror (DM)-based adaptive optics $(\mathrm{AO})$ with a confocal scanning laser ophthalmoscope. A low-cost bimorph DM with a large stroke of $50 \mu \mathrm{m}$ and an aperture of $20 \mathrm{~mm}$ was utilized to realize a strategy for successive AO control of aberration correction, which permitted open-loop compensation for low-order aberrations and closed-loop correction of high-order aberrations to acceptable root mean square errors of $<0.08 \mu \mathrm{m}$ in all subjects. Spherical mirrors were folded in a nonplanar configuration to minimize off-axis aberrations and provide a compact, cost-effective design, which achieved a diffraction-limited performance capable of imaging individual photoreceptor cells and blood vessels not only in healthy subjects but also in patients suffering from retinitis pigmentosa. The adaptive optics scanning laser ophthalmoscope (AOSLO) images of the diseased retina had much higher resolutions than those captured by the commercial AO fundus camera, and loss of the photoreceptor mosaic could be distinguished more accurately due to the improvement in resolution. The compact design and easy handling of the bimorph DM-based AO control may facilitate the translation of AOSLO into clinical settings, and this prototype development will continue with future device refinement and extensive clinical testing. $\odot$ The Authors. Published by SPIE under a Creative Commons Attribution 4.0 Unported License. Distribution or reproduction of this work in whole or in part requires full attribution of the original publication, including its DOI. [DOI: 10.1117/1.NPh.6.4.041111]
\end{abstract}

Keywords: ophthalmic optics; adaptive optics; confocal microscopy; optical engineering; medical optics instrumentation.

Paper 19053SSRR received May 31, 2019; accepted for publication Oct. 14, 2019; published online Nov. 5, 2019.

\section{Introduction}

The adaptive optics scanning laser ophthalmoscope (AOSLO) integrates adaptive optics $(\mathrm{AO})$ with the scanning laser ophthalmoscope to obtain retina images with cellular and subcellular resolutions in vivo. ${ }^{1-3}$ This device is now in widespread laboratory use for a growing range of research topics. ${ }^{4-7}$ In recent years, AOSLO has shown promise as a sensitive diagnostic tool, ${ }^{8-10}$ such as for blinding retinal diseases, which may be related to photoreceptors in the retina. ${ }^{11}$

One key element in an AO system is the wavefront compensating device, which plays the role of realizing corrections of aberrations. Many kinds of wavefront compensating devices have been widely used, such as liquid crystal phase modulators, ${ }^{12,13}$ microelectromechanical system (MEMS)-based deformable mirrors (DMs), ${ }^{14-16}$ and piezoelectric DM. ${ }^{17-19}$ However, these devices have a limited dynamic range (around 1 to $8 \mu \mathrm{m}$ stroke), which is not enough to correct human eye aberrations, especially for diseased eyes, which have much larger aberrations. ${ }^{20,21}$ Electromagnetic DM with a stroke of 20 to $60 \mu \mathrm{m}$ has proved to be enough for the correction of human ocular aberrations, ${ }^{22-24}$ but its high cost and lower flatness reduce its fidelity and wide use.

\footnotetext{
*Address all correspondence to Yi He, E-mail: heyi@ sibet.ac.cn; Guohua Shi, E-mail: ghshi_lab@126.com

tThese authors contributed to the work equally and should be considered
} co-first authors.
Therefore, there are two barriers to the widespread use of AOSLO by clinicians and research scientists. The first barrier is the high cost of DMs with larger strokes, which will likely remain relatively high even if the DM cost decreases over time as a result of technological innovation and commercialization. The second obstacle is that the system complexity slows the translation of AOSLO from the research lab to the clinic. To promote the compensation capacity of aberrations, especially for diseased eyes with large amplitude aberrations, dual DMs have been utilized at the cost of system complexity. ${ }^{25}$ Currently, the AOSLO systems designed and constructed in the lab for the best imaging performance can be operated only by researchers with extensive expertise in optics. Hence, the current system design is not feasible for use in clinical practice due to its cost, complexity, size, and inconvenience. ${ }^{26-30}$

To reduce these barriers, we customize a bimorph DM with 35 actuators in an aperture of $20 \mathrm{~mm}$, consisting of a piezoceramic disk bonded to a rigid substrate to produce a maximal stroke of more than $50 \mu \mathrm{m}$. Moreover, this design is one of the most cost-effective DMs to date. ${ }^{31,32}$ Integrated with this bimorph DM-based AO, we designed and built an AOSLO instrument for a clinical setting with the goal of being applicable to as many research and clinical applications as possible. First, the optical component was designed to span a 3-deg field of view (FOV) with diffraction-limited resolution. Second, a mechanical tolerance analysis was performed to design all mechanical components to minimize the instrument complexity and size. Third, the electronic control system for the AO closed-loop aberration 
correction and retina image acquisition was designed through the implementation of only one computer to improve the ease of use. Last, the AOSLO prototype was tested in healthy volunteers. The results demonstrate that the prototype is capable of reducing ocular aberrations with large amplitudes, and it is the first device to provide high-resolution images of the retina in subjects with retinitis pigmentosa (RP) to accurately distinguish loss of the photoreceptor mosaic.

\section{Materials and Method}

\subsection{Optical Design}

The AOSLO system has a double-pass optical path, including a light source, horizontal and vertical scanners, a wavefront sensor, a DM, and a photomultiplier tube (PMT) detector. Note that the wavefront sensor and PMT detector are on-axis optical modules that are relatively free from aberrations. Only the light delivery path is optically designed and optimized using ZEMAX (Radiant ZEMAX LLC, Redmond, Washington), and an overview of the optimized optical design is shown in Fig. 1. Spherical mirrors in an off-axis arrangement are used to relay the planes conjugate to the pupil of the eye to avoid backreflected light, and these mirrors are used in pairs, creating an afocal telescope to preserve conjugate planes. To form a rectangular imaging raster on the retina, a resonant scanner (SC30-3 $\times 4$, Electro-Optical Products Corp) line-scanned the retina horizontally at $16 \mathrm{kHz}$ with an optical aperture of $3 \mathrm{~mm}$, and a slow galvometric scanner (6800HP, Cambridge Tech. Corp) scanned the vertical direction at $30 \mathrm{~Hz}$.

A 670-nm superluminescent diode (SLD) with 10-nm bandwidth (SLD-261-HP, Superlum, Ireland) was used for wavefront sensing and retina imaging after being collimated to produce parallel lights with a diameter of $\sim 6.0 \mathrm{~mm}$ through the collimating lens L1. The delivery optical path consists of four 4- $f$, afocal telescope pairs that relay the eye pupil to several pupil planes, including the planes of the DM, horizontal and vertical scanners, and wavefront sensor. The DM is placed at the pupil conjugate closest to the light source to avoid ray light movements or wanders in the DM surface. All the afocal telescopes of spherical mirrors are ordered to achieve matches between each pupil aperture. Since the off-axis mirrors are likely to generate off-axis aberrations such as astigmatism and coma, a straightforward way to reduce the off-axis angle and astigmatism is to use spherical mirrors with long focal lengths, but this increases the overall size of the device. By folding spherical mirrors in a nonplanar configuration and alternating the angle of beam reflection of these mirrors from the horizontal and vertical directions, offaxis aberrations can be minimized. ${ }^{28}$ Therefore, spherical mirrors M5 and M6 were carefully used off the optical plane to compensate astigmatism aberrations, and folding angles were optimized (under constraints for ray clearance and mechanical mount clearance) on both the horizontal and vertical directions to minimize monochromatic aberrations in the retina.

In addition, the spherical mirror M8 prior to the eye consisted of 25.4-mm-diameter lenses with an effective focal length of $160 \mathrm{~mm}$. This focal length was chosen to give a working distance of $\sim 30 \mathrm{~mm}$ and have sufficient room for the plano mirror PM2 to fold the light path for easy retina imaging. Limited by constrains of XY scanners, a $3 \mathrm{deg} \times 3 \mathrm{deg}$ FOV was used to design the AOSLO. However, the real FOV for retina imaging should be cropped smaller than 2 deg, suffering from the human eye's isoplanatic angle in an AO system.
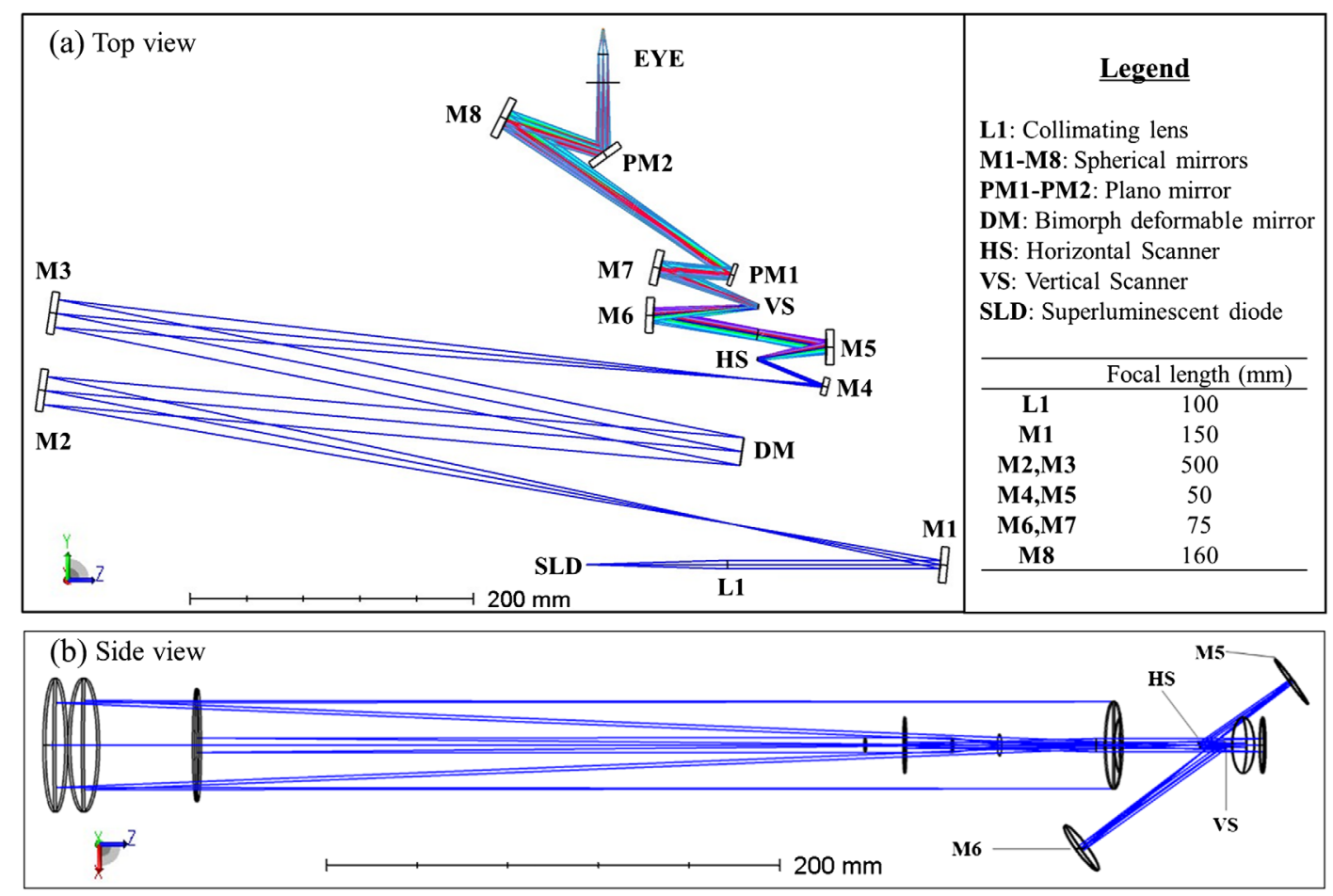

Fig. 1 (a) A top view schematic of the AOSLO optical design. Note that optical components are folded in three dimensions and is flattened for illustration. All optical components are labeled and described in the legend. The optical design is the light delivery path excluding the on-axis optical components free from aberrations. (b) The light delivery path in side view as shown in Fig. 1(a). M5 and M6 are arranged out of the main optical plane to compensate for astigmatism. 
After the initial optical structure was chosen, the optical design was optimized by minimizing spot sizes on the retina for 17 uniformly distributed points spanning the FOV and for three different vergences $(-5 \mathrm{D}, 0 \mathrm{D}$, and $+5 \mathrm{D})$. Taking a modified Gullstrand eye model, ${ }^{33}$ diffraction-limited performance for a vergence range of $10 \mathrm{D}$ over all the FOVs has been achieved for high-resolution AO imaging, and the optical optimization performance is shown in Fig. 2. Note that all spot diagrams were calculated on the focal plane of the model human eye, assuming an $\mathrm{AO}$ correction of low-order aberrations when a seventh-order Zernike polynomial was adopted to estimate the wavefront aberrations in ZEMAX. For an emmetropia eye, much better performance can be expected that the residual wavefront root mean square (RMS) was lower than $\lambda / 21$. With the increase of vergence, the flatness of $\mathrm{AO}$ corrections would decrease for myopic and hyperopic eyes with low-order aberrations, and the residual wavefront RMS of astigmatism would slightly increase while still meeting the diffraction limit requirement. Benefitting from the large stroke of the bimorph DM to accomplish AO aberration corrections, the residual wavefront RMS at $670 \mathrm{~nm}$ was lower than $\lambda / 14$ for all vergences.

Since the horizontal and vertical scanners move to form the two-dimensional (2-D) imaging raster at the retina plane, measures must be taken to ensure that the chief ray pivots around a stationary point in the center of all the pupil planes in this AOSLO system, including the two scanners and the eye pupil.
Figure 3 shows light maps on the three pupil planes. Along the light passing to the retina, the aberration is growing to cause the displacement of the chief ray on those pupil planes, and the maximum beam wander for all the FOVs was $\sim 6.25 \%$ and occurred on the eye pupil. Actually, beam wander in the pupil planes is bound to occur due to the mirror-based reflective optical system with beam scanning, but this is acceptable considering that human pupils can usually be dilated to 7 to $8 \mathrm{~mm}$, and the beam wander will decrease once the scanning FOV is appropriately reduced.

For those on-axis optical components, a custom-made Hartmann-Shack wavefront sensor (HSWS) consisting of a square lenslet array with a focal length of 5 and $0.2 \mathrm{~mm} \times$ $0.2 \mathrm{~mm}$ pitches was used for wavefront sensing. To complete the double-pass optical path, light backscattered from the retina passed through the delivery path and was split by several beam splitters (not shown in this figure), of which an 80/20 beam splitter was utilized to separate the illuminating and imaging beam. Then a 95/5-beam splitter was ordered to separate the detecting and wavefront sensing beams. The detecting beam was collected using PMTs (H7422-20, Hamamatsu, Japan) and then focused by an achromatic lens with a focal length of $200 \mathrm{~mm}$. For the confocal configuration, a pinhole with a diameter of $50 \mu \mathrm{m}$ equal to $\sim 1.44$ times the Airy disk diameter was set in front of the PMT, which has been demonstrated to give a good balance between image sharpness and throughput. ${ }^{34,35}$

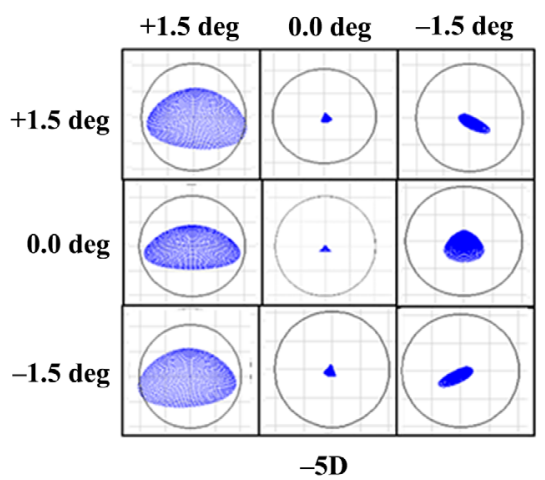

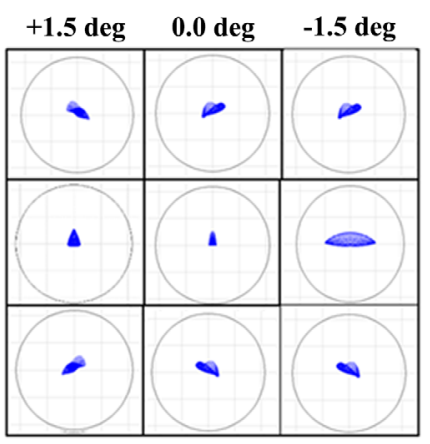

OD

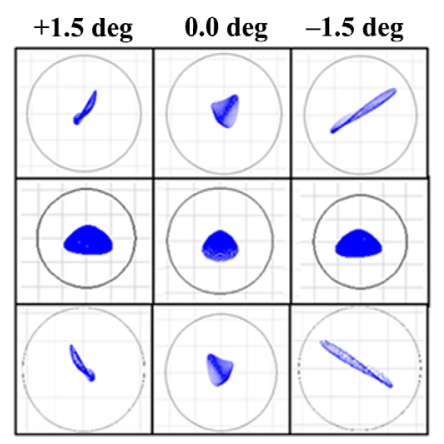

$+5 \mathrm{D}$

Fig. 2 Spot diagrams for nine typical configurations evaluated on the retina over a 3 deg $\times 3$ deg FOV for a vergence range of $10 \mathrm{D}$ in the AOSLO optical design. Configurations are grouped by vergences, and all configurations are diffraction limited for the wavelength of $670 \mathrm{~nm}$ with a pupil diameter of $6.4 \mathrm{~mm}$. The radius of Airy disk (black circle) is $2.09 \mu \mathrm{m}$.
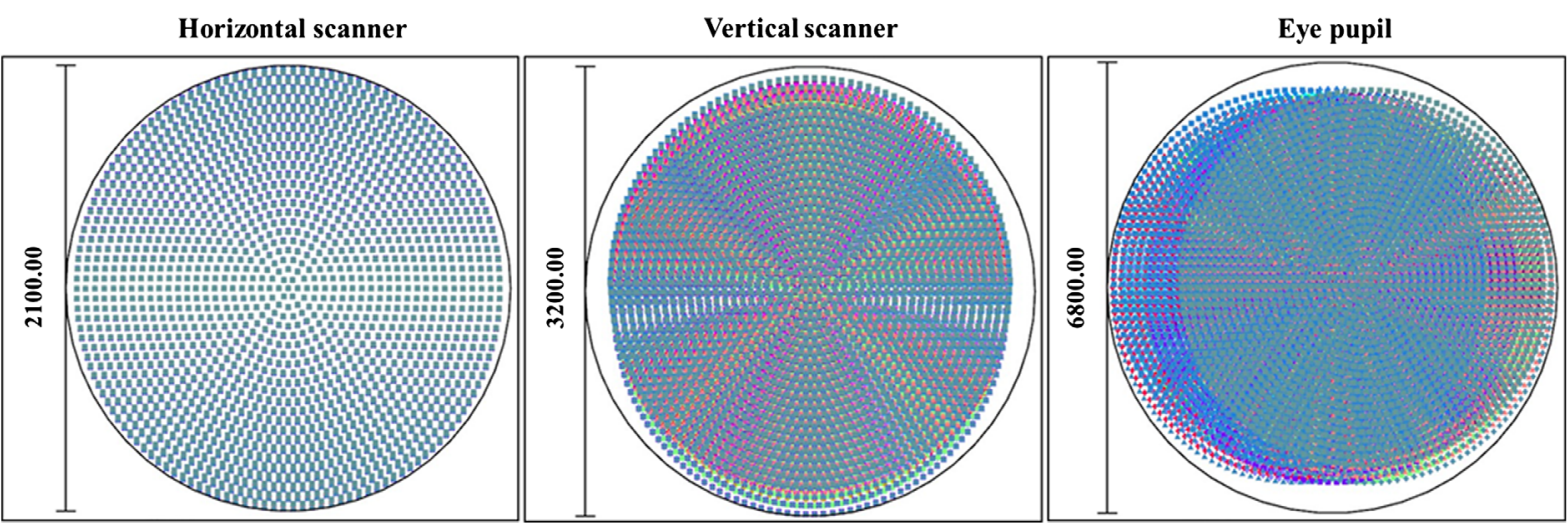

Fig. 3 Light maps for the three pupil planes over all the FOVs, and different scanning FOVs are coded by color. Black circle represents the maximum diameter occupying all the wandered rays. 


\subsection{Adaptive Optics Control and Electronic Design}

Compared to other widely used clinical SLO devices, AO for aberration correction was integrated with the SLO module in an increase of complexity manner, thereby making it difficult to operate on separate computers allowing for aberration correction, scanning timing, and retina imaging. ${ }^{36}$ Therefore, we optimized the AO control and electronic design to realize an easy-to-use AOSLO system for aberration correction and retina imaging.

In a typical implementation of a wavefront sensor-based $\mathrm{AO}$ system for human retina imaging, the limited stroke of DM made it difficult to accurately compensate for ocular aberrations, and additional trial lenses were introduced to precompensate low-order aberrations with large amplitudes. ${ }^{37,38}$ The DM played the role of high-order aberration correction, which in general requires lower stroke. However, this approach requires manually changing the graduation of trial lenses for each individual subject, which can be a tedious and complex process ${ }^{28,39}$ and can even disturb the $\mathrm{AO}$ aberration correction once the trial lens is located away from the pupil conjugate. In this study, with the developed bimorph DM of large stroke values (up to $50 \mu \mathrm{m}$ ), we propose a successive $\mathrm{AO}$ control strategy to compensate ocular aberrations without additional trial lenses, which is a bit similar to the strategy of the woofer-tweeter dual DM AOSLO. ${ }^{40}$ However, only the single bimorph DM, rather than two DMs, is utilized for lower- and higher-order aberration corrections. In practice, the ocular aberrations are measured through the HSWS, and efficiently partitioned to the two parts of low-order and high-order aberrations in the process of wavefront restorations. Therefore, the bimorph DM is predistorted in an openloop mode to match the expected wavefront of low-order aberration correction, in which all actuators subjected to the same voltage will produce a defocus phase, and different voltages on the fan-shaped actuators can produce an astigmatism phase. Once the open-loop AO correction of low-order aberrations works well after two to five iterations ( 20 to $50 \mathrm{~ms})$, the closed-loop AO control is engaged to correct the high-order aberrations.

In the electronic module of the AOSLO system, the HSWS images were captured with a speed of $120 \mathrm{~Hz}$ using a GigE camera (Manta G-235, Allied Vision, Germany), and then AO control signals were calculated and updated in sequence, including wavefront errors, aberration deflection maps, Zernike coefficients, and the DM compensation signals. When the desired focus of the retina is achieved by open-loop correction of low-order aberrations, such as near the plane of the photoreceptors, the closed-loop AO control is used to minimize the wavefront errors.

When the AO aberration correction works well and is locked, an independent real-time controller (custom-built field-programmable gate array) generates driving signals and synchronous position signals for the horizontal and vertical scanners, and start-of-frame synchronization signals are also provided to the frame grabber (PCIe-1433, NI) to capture retinal high-resolution images from the PMT detector. Unlike other AO retinal imaging systems that require separate computers to control different subsystems, ${ }^{36,38}$ only one computer is needed to implement the two electronic control modules in the proposed AOSLO system, which is shown in Fig. 4. Retinal images in AVI image files with a speed of 30 frames per second can be recorded and stored in the database of the computer, and image processing, such as montaging, is performed offline in MATLAB (MathWorks, Inc.).

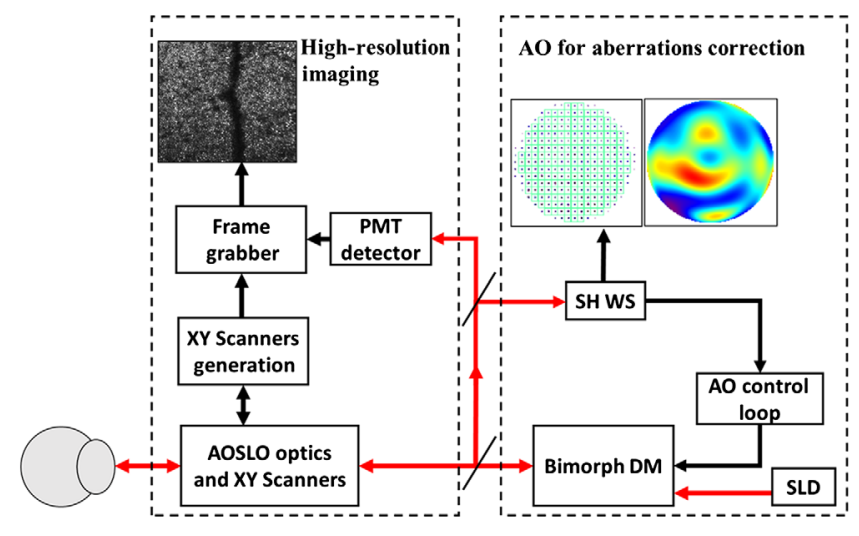

Fig. 4 The electronic control of the AOSLO system. Dashed lines represent the two major control subsystems, the high-resolution imaging (left), and the $\mathrm{AO}$ for aberration correction (right). Red lines with arrows present the optical signals, and the black lines present the electronic signals.

\subsection{Mechanical Design}

To reduce the complexity of constructing an AOSLO system, the mechanical design for the system was developed in SolidWorks (Dassault Systèmes SolidWorks Corp., Concord, Massachusetts), as shown in Fig. 5(a). A brief rule is that all the optomechanical components are set on a thick bottom plate, and all the other components such as electronic devices are included in the lower part of the system for weighting balance. According to the maximum permissible positional errors of optical design, a mechanical tolerance analysis was performed to determine the adjustments of each mirror and lens to optimize the assembly, and then all the mirror mounts, lens tubes, and lens spacers were designed and fabricated to accommodate the closely spaced optics of the system and minimize its footprint. Except for the bottom plate made of thickened iron, the internal skeleton and other structural components were made of aluminum to simplify fabrication and maintain low weight. To optimize performance between the assembly and operation, we used the minimum number of adjustments in the mechanical design, including the adjustability of the confocal pinhole, horizontal and vertical scanners, HSWS, and DM. The confocal pinhole was mounted on a linear motion stage with total travel lengths of $25 \mathrm{~mm}$ to allow adjustments in the $X Y Z$ direction. The horizontal and vertical scanners were mounted via a mechanical base with curved slots to adjust the angular orientation while maintaining the correct center of rotation. The HSWS and DM were constructed with three pull/push structures, allowing the tilt/pitch of the laser beam to be adjusted by $3 \mathrm{~mm}$ and $15 \mathrm{deg}$. The outer casing was made of iron with painting and chamfering, and the fabricated setup is shown in Fig. 5(b). A chin rest was mounted into the bottom plate using $X Y Z$ translation stages for light beam alignment. The AOSLO prototype weighed $55 \mathrm{~kg}$ and was $80-\mathrm{cm}$ long $\times 60$-cm wide $\times 120$-cm tall. However, the AOLSO mainframe had a much smaller size of 80 -cm long $\times 60$-cm wide $\times$ $20-\mathrm{cm}$ tall and a lighter weight of $18.5 \mathrm{~kg}$ when all other electronic devices were moved to an additional box.

\section{Results}

\subsection{Adaptive Optics Performance}

Prior to retina imaging, all human subjects were fully informed of the procedures and consequences of the study, and all subjects 


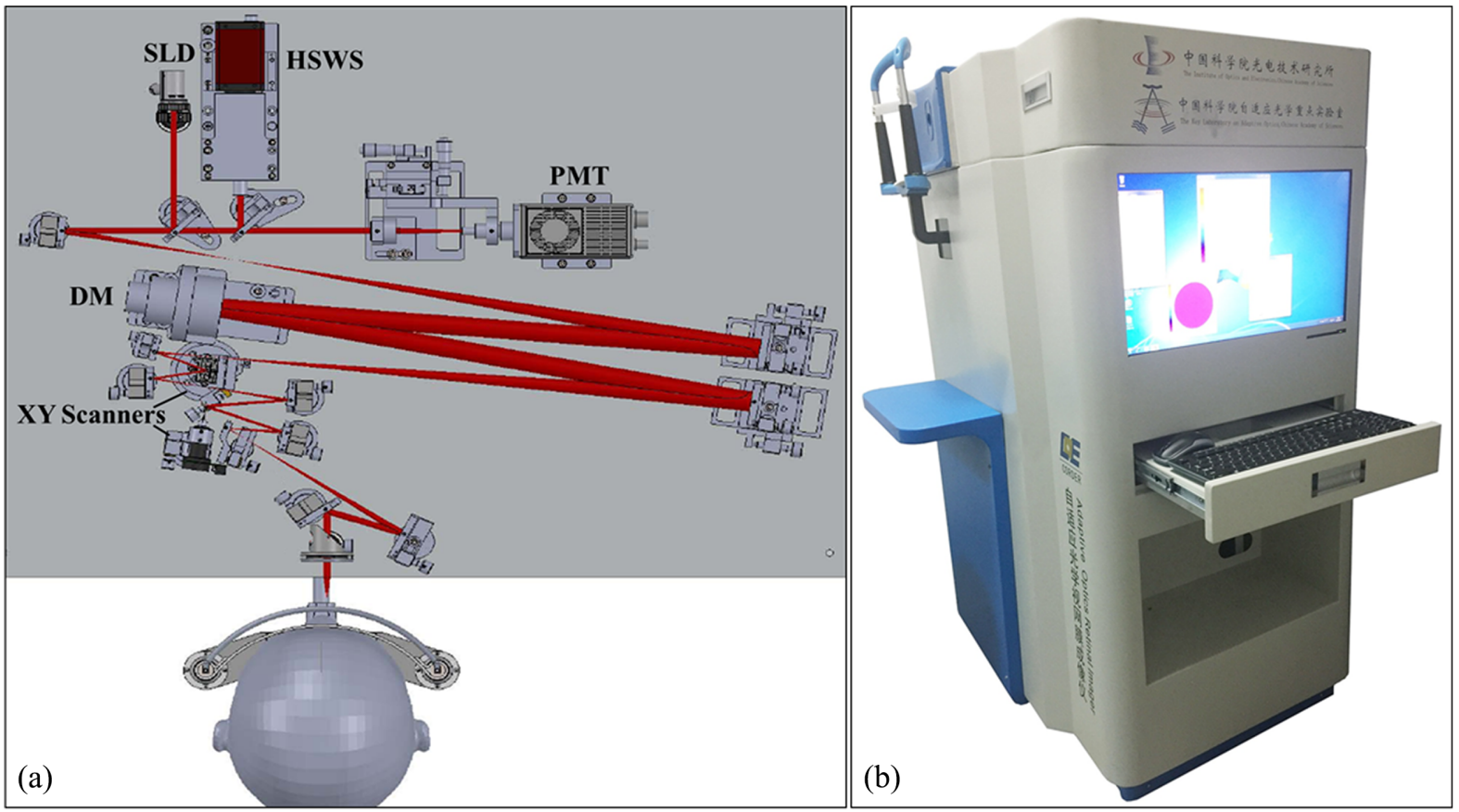

Fig. 5 The mechanical design of the AOSLO system in top view (a) showing the internal optical components. (b) Photograph of the fabricated setup with case.

provided signed informed consent. Eyes to be imaged were dilated with $0.5 \%$ tropicamide to 6- to 7 -mm pupils and illuminated by the SLD laser beam always kept below $140 \mu \mathrm{W}$, matching safety standards of the American National Standards Institute maximum laser exposure in more than one order of magnitude, even if the exposure time lasts almost 50 min. ${ }^{41,42}$ In practice, each single imaging experiment can be only executed for a period of no more than $3 \mathrm{~min}$ or even shorter time, due to eye motion or blink.

Subjects had refractive errors up to $6.5 \mathrm{D}$, with lower-order aberrations of defocus and astigmatism. Such large, lower-order aberrations can lead to the failure of closed-loop control, especially since the large defocus aberrations take many cycles to be corrected while causing the correction to oscillate. To prevent the AO aberration correction from failing, the bimorph DM was initiated first and run in open-loop mode, where it corrected the system and subject defocus aberrations through a fixed and static aberration correction until the wavefront aberration RMS was reduced to below $1.0 \mu \mathrm{m}$, ensuring that the HSWS sensor was within its working range at the start of the closed-loop correction for high-order aberrations. Then the HSWS was activated to measure high-order aberrations, and the DM was left in closed-loop mode for a dynamic AO correction until the wavefront RMS was decreased to a diffraction-limit performance.

The estimation of the wavefront aberrations was fitted to a seventh-order Zernike polynomial, using the normalization given by the OSA standard for ocular aberrations. ${ }^{43,44}$ Figure 6 shows an example of the AO performance achieved in one healthy human subject (male, age 32, pupil with a diameter of $\sim 6.42 \mathrm{~mm}$ ). In Figs. 6(a)-6(c), color-coded aberrations maps are illustrated for three cases: initial aberrations without AO correction, aberrations with open-loop correction, and aberrations with closed-loop correction. The averaged aberrations separated by Zernike order and wavefront RMS reduction course with the AO correction are also shown in Figs. 6(d) and 6(e). Note that the initial aberrations presented typical myopia $(\sim 2 \mathrm{D})$ and astigmatism ( 1D) and a rather irregular cornea that introduced some high-order aberrations. With open-loop AO correction, the average RMS error improved from 2.16 to $0.77 \mu \mathrm{m}$, mostly reducing low-order aberrations. After the closed-loop AO correction, the RMS errors generally reduced to $0.034 \mu \mathrm{m}$. The improvement in all Zernike orders is shown in Fig. 6(d), although the most improvement was seen in the first six orders. In Fig. 6(e), the $10 \%$ to $90 \%$ rise-time for the AO correction was $\sim 100 \mathrm{~ms}$, which corresponds to a temporal bandwidth of $\sim 10 \mathrm{~Hz}$ for the AO correction duration.

The AO performance was further studied in a small-scale population experiment. A total of 20 subjects between the ages of 20 to 40 years (average: 32 years; standard deviation: 6 years) were recruited for the experiment. Figure 7 shows the RMS wavefront errors with and without AO corrections. All the subjects were corrected to better than $0.08 \mu \mathrm{m}$ RMS errors. The average RMS error improved from $3.05 \pm 1.17$ to $0.067 \pm$ $0.012 \mu \mathrm{m}$ with $\mathrm{AO}$ correction.

\subsection{Photoreceptor Imaging}

Since the bimorph DM-based AO was effective in correcting human ocular aberrations, Figs. 8(a) and 8(b) compare retina images without and with $\mathrm{AO}$ corrections for a female healthy subject, aged 26 years with a $\Phi 6.84-\mathrm{mm}$ pupil. Both singleframe images contained 512 pixels $\times 449$ pixels of the same retina location $1.5 \mathrm{deg}$ from the fovea center. With an open-loop correction of relatively large low-order aberrations ( $-4 \mathrm{D}$ defocus and $-1.5 \mathrm{D} / 065$ astigmatism), even the best-focus image in Fig. 8(a) cannot distinguish any retinal photoreceptors. When the closed-loop AO correction was activated to dynamically correct ocular aberrations, an image of the same subject and retinal location was shown in Fig. 8(b), and photoreceptor cones can be clearly resolved due to the increased brightness, enhanced contrast, and improved resolution. With AO correction in Fig. 8(b), the ocular wavefront error was corrected to the RMS value of 


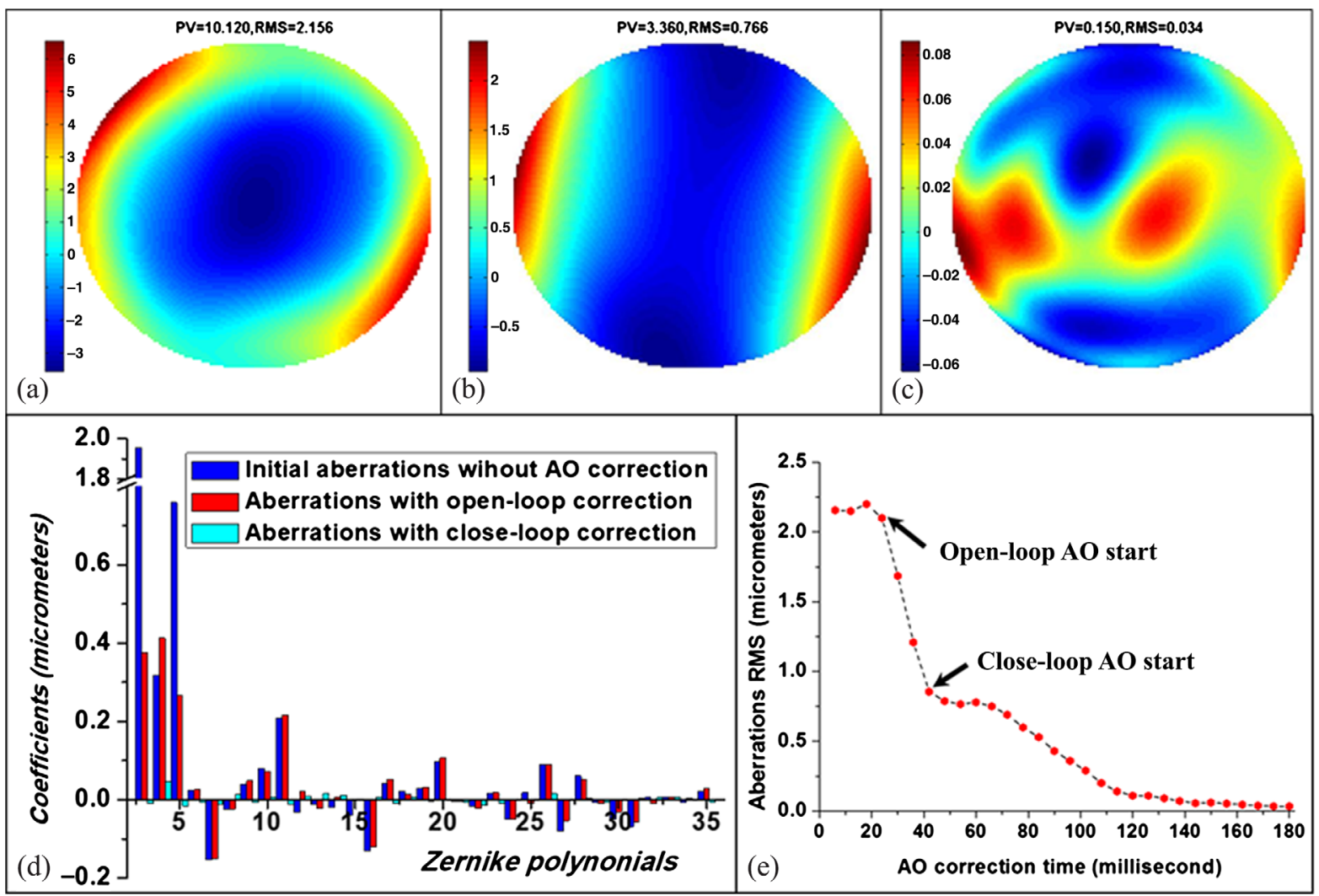

Fig. 6 Example of the AO performance for one healthy subject with a $6.42-\mathrm{mm}$ pupil. Wavefront aberration maps in (a)-(c) represent the initial aberrations, aberrations with open-loop correction, and aberrations with closed-loop correction, respectively. The bar diagram in (d) shows the aberration coefficients in Zernike orders, and the temporal profile in (e) shows the RMS wavefront error reduction during the whole AO correction.

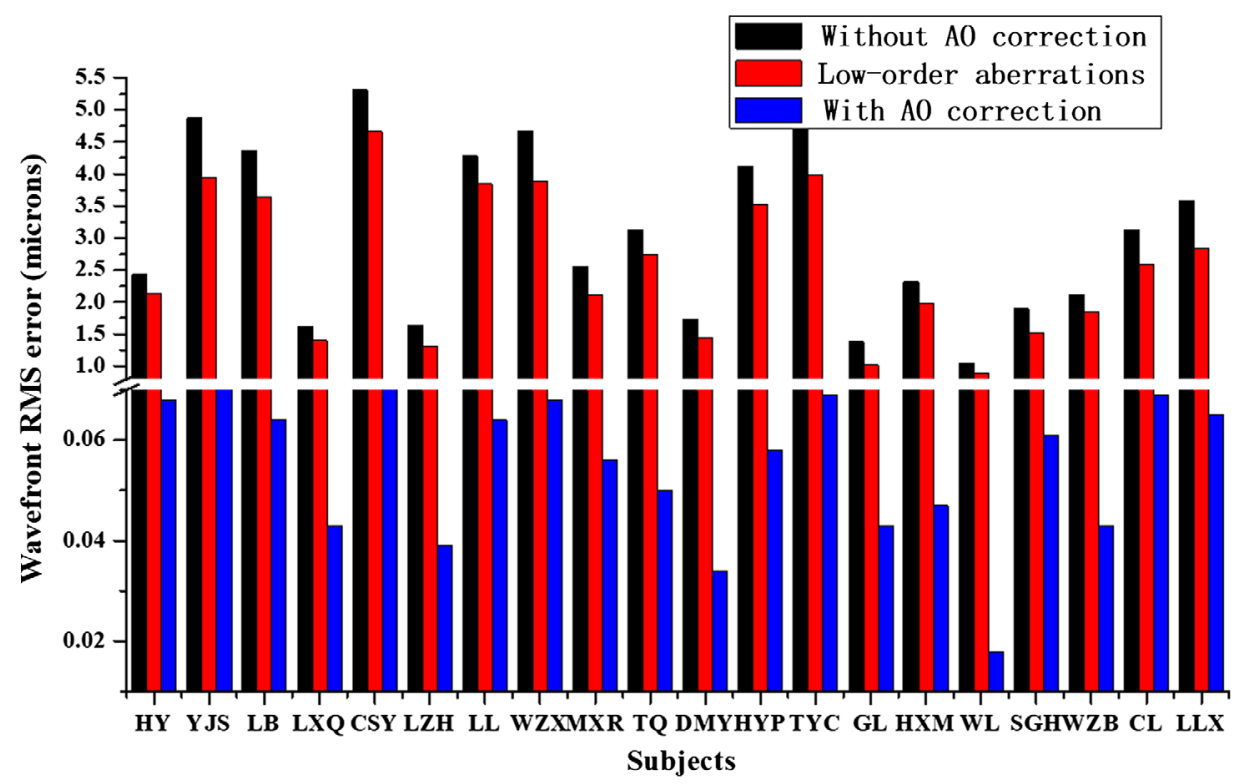

Fig. 7 Aberration RMS errors of 20 subjects without and with $\mathrm{AO}$ corrections in black color bar and blue color bar, respectively. Low-order aberrations are illustrated in red color bar.

about $0.046 \mu \mathrm{m}$, and much sharper structural details of the retina, such as photoreceptors, can be clearly distinguished.

The power spectra of retina images are presented to demonstrate the improvement of AO correction in Fig. 8(c), where the
Fourier spectrum for each frequency is radially integrated. Benefitting from the $\mathrm{AO}$ correction of human ocular aberrations, the power spectra increased at low frequencies and decreased at high frequencies. This indicates that the image resolution 

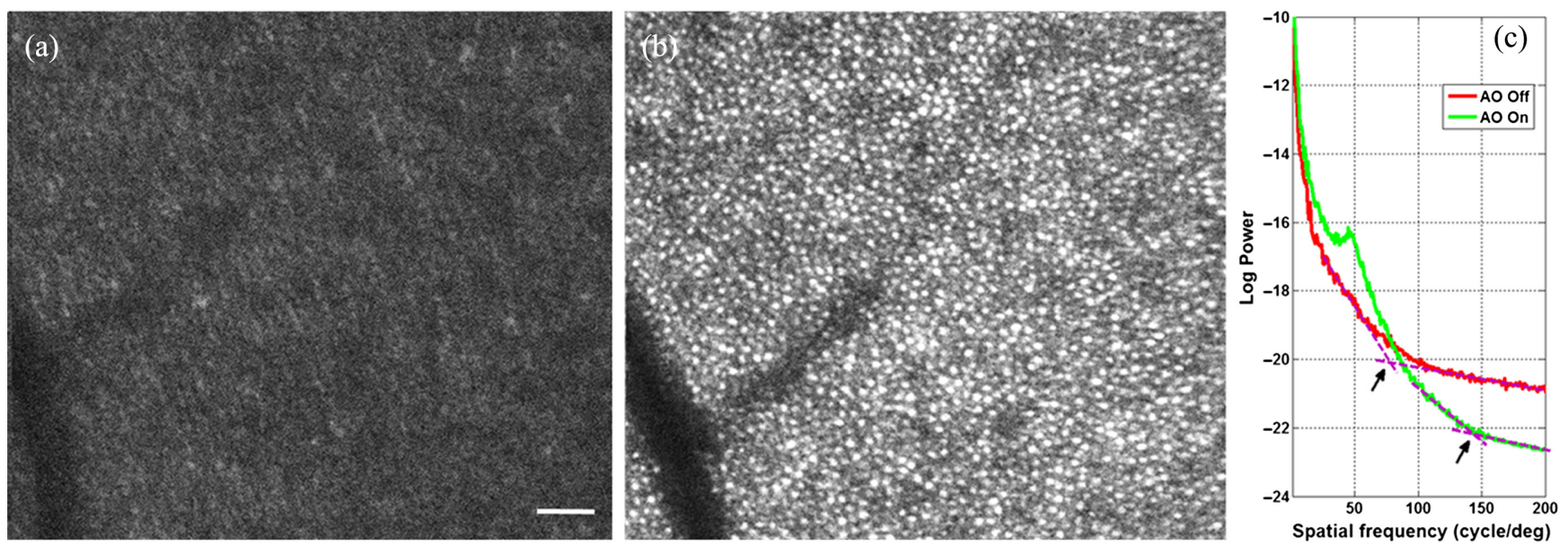

Fig. 8 Comparison of retina images (a) without and (b) with AO closed-loop corrections. (c) The spectra power plot for retina images. The purple dot-dash lines were added to show the trends of the power spectra curves. All single-frame images are 512 pixels $\times 449$ pixels, and the retina image without $A O$ has been scaled in intensity $2 \times$ relative to the image with $\mathrm{AO}$ correction. Both images are taken from the same retina location $1.5 \mathrm{deg}$ from the fovea center, and the FOV subtends $1.7 \mathrm{deg} \times 1.5 \mathrm{deg}$. The scale bar is $50 \mu \mathrm{m}$. Movie sequence of $30 \mathrm{fps}$ is also presented to show the AO correction procedure (Video 1, 2.75 MB, mp4 [URL: https://doi.org/10.1117/1.NPh.6.4.041111.1]).

corresponding to low frequencies was improved, and the noises corresponding to high frequencies were suppressed.

For comparison in this power spectra, the highest resolvable spatial frequency of the retina image was about 75 cycles/deg without AO correction, and it increased to 140 cycles/deg with AO correction. The spatial frequency around the Yellot's ring ${ }^{45}$ determines the highest resolvable spatial frequency. Highlighted by an arrow in Fig. 8(c), the radius of the Yellot's ring is about 140 cycles/deg, which is relatively close to the highest resolvable frequency with AO correction. According to the Raleigh criteria, the minimum resolution for a $\Phi 6.84-\mathrm{mm}$ pupil at the wavelength of $670 \mathrm{~nm}$ is about $2.03 \mu \mathrm{m}$ ( 145 cycles/deg in spatial frequency) for a modified Gullstrand eye model, ${ }^{33}$ so the retina image with $\mathrm{AO}$ correction is very close to but still below the Raleigh diffraction limit, suffering from the system noise of intensity conditions.

\subsection{Axial Sectioning and Imaging}

With the bimorph DM of large strokes of $\sim 50 \mu \mathrm{m}$, ocular aberrations can be corrected to achieve the diffraction-limit resolution, and enough strokes remain to allow for axial sectioning of the retina. When the $\mathrm{AO}$ correction is accomplished to achieve a wavefront error RMS of $<0.1 \mu \mathrm{m}$ (can be thought as a successful aberration compensation in most situations), an additional bias to the defocus coefficient is applied to the bimorph DM, and light beam will be focused at different retina depth. Benefited from the confocal pinhole, only light from near the plane of best focus gets imaged, and true axial sectioning of the retina can be obtained. Figure 9 shows retinal images acquired at several depths while continuously changing the DM sectioning depth, which have the same location in the subject of Fig. 8. The focus was initially at the photoreceptor layer in Fig. 9(a), and then sectioned to the blood vessel layer in Fig. 9(c) and the nerve fiber layer in Fig. 9(d). Therefore, three layers of the retina section can be clearly revealed in Fig. 9, which entirely spans $\sim 300 \mu \mathrm{m}$ of the axial depth in this retina. For practical purposes, retinal image between the photoreceptor layer and the blood vessel layer is also presented in Fig. 9, where the photoreceptor mosaic cells and the blood vessel are recognizable in Fig. 9(b).

\subsection{Retinitis Pigmentosa Disease Imaging}

The proposed AOSLO prototype was then used to image retinal patients with RP. RP is a genetic disorder of the eyes in which pigments gradually form in the retina, thereby causing the progressive loss of rod photoreceptor cells and further loss of cone photoreceptor cells, eventually resulting in severe vision loss. ${ }^{46,47}$ Unfortunately, the loss of photoreceptor cells cannot be imaged by commercially available retinal ophthalmoscope devices due to lack of resolution, and even the most extensive examinations for RP diagnosis, including electroretinograms, visual field testing, and genetic testing, cannot image photoreceptor loss directly. ${ }^{47}$ With the help of the latest commercial AO device, the AO-fundus camera (RTX1, Orsay, France), clinicians can diagnose RP for the first time by observing the absence of photoreceptor cells. Figure 10 shows a representative AO image of a patient with RP (aged 20 years, female). The high-resolution AO-fundus image in Fig. 10(a) shows an abnormality on the photoreceptor morphology [oculus dexter (OD), recorded at location: temporal $=4.0 \mathrm{deg}$; superior $=3.0 \mathrm{deg}$, and the AOSLO images of this subject at the same location are presented in Figs. 10(d) and 10(e).

High-resolution images in Fig. 10(a) appear sharp with AO correction, and an abnormality on the photoreceptor morphology can be observed, in which the photoreceptor loss can be noted compared to the normal retina. The enlarged regions of interest in Figs. 10(b) and 10(c) indicated with the red squares in Fig. 10(a) show areas of photoreceptor loss at those locations (cf. overlaid manual segmentation). However, photoreceptor mosaic is slightly blurred, and the vessel edge is particularly blurred in Figs. 10(a)-10(c). The AOSLO images in Figs. 10(d) and 10(e) avoid photoreceptor blurriness, clearly showing the area of photoreceptor mosaic loss (red-labeled areas). Actually, photoreceptor loss areas in Figs. 10(d) and 10(e) are smaller and less than those in Figs. 10(b) and 10(c), and more accurate 

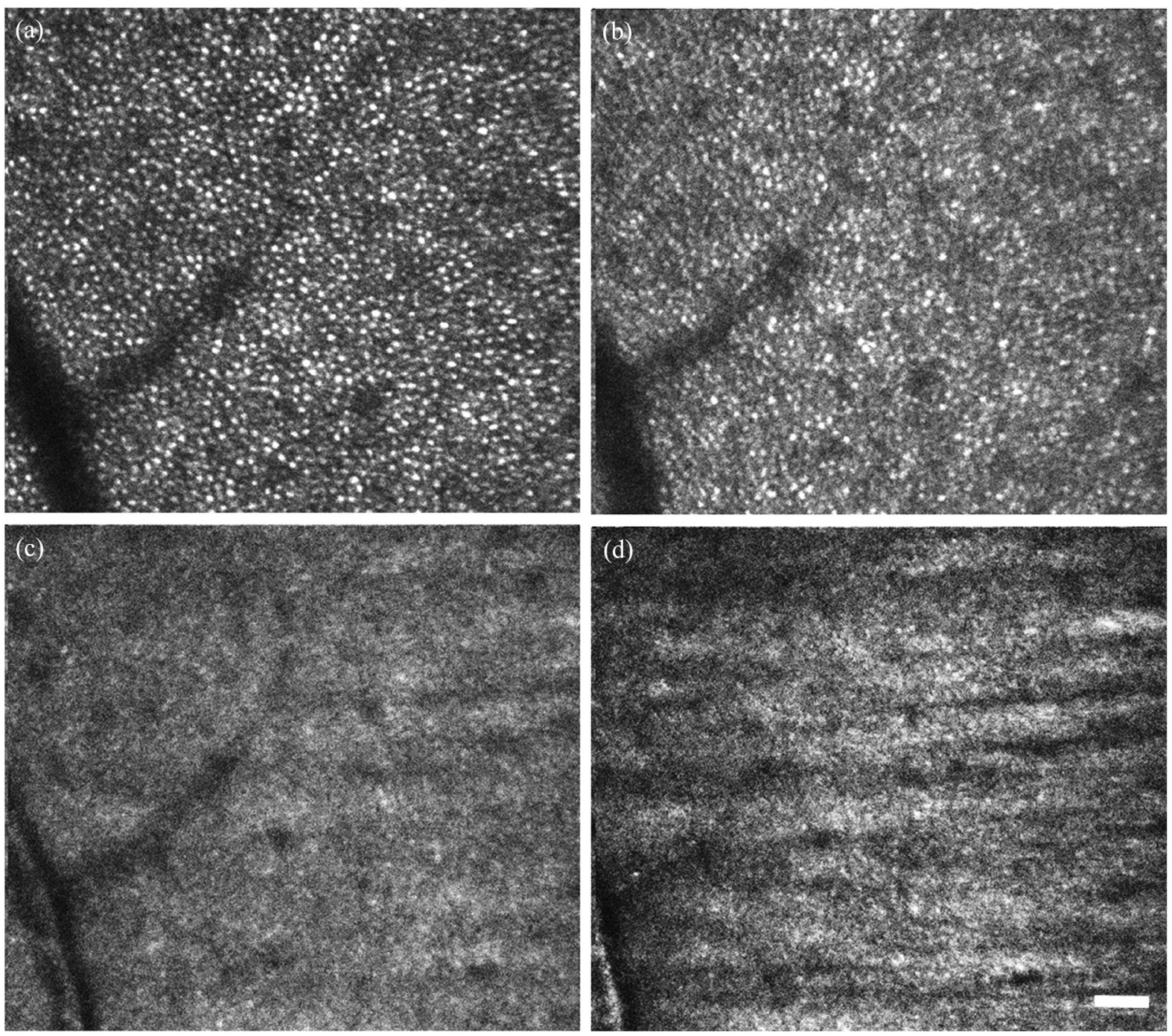

Fig. 9 Axial sectioning and imaging at different retinal depths for the same location as in the subject of Fig. 8. (a)-(d) The light beam is focused at different surface of the retina while continuously changing the DM sectioning depth. (a) Layers of photoreceptor, (c) blood vessel, and (d) nerve fiber layer are clearly distinguished, and retinal image between the photoreceptor layer and the blood vessel layer is also presented in (b). Scale bar is $50 \mu \mathrm{m}$. Movie sequence of $30 \mathrm{fps}$ is also presented to show the axial sectioning procedure (Video 2, $13.9 \mathrm{MB}, \mathrm{mp4}$ [URL: https://doi.org/10.1117/1.NPh.6.4.041111.2]).

photoreceptor density and spacing can be concluded in AOSLO images. In Fig. 10(d), the photoreceptor blurriness affected the shape of vessels, causing obscure vascular boundaries. Much more photoreceptor blurriness can be observed in Figs. 10(b) and 10(c), and some are overlaid on vessels (yellow-labeled areas), probably leading to incorrect observations of blood vessels. Correspondingly, there is almost no photoreceptor blurriness in Figs. 10(d) and 10(e), and an apparent bifurcation of vessels can be distinguished clearly, which is mistaken as visible photoreceptor loss in Fig. 10(c).

The Fourier spectral power plots of this subject are shown in Fig. 11. For the AO-fundus images of this subject in Figs. 10(b) and 10(c), the highest resolvable frequencies were about 90 and 95 cycles/deg, respectively. According to the Raleigh criteria, the theoretical minimum resolvable spatial frequency for a $\Phi-6.62-\mathrm{mm}$ pupil at the wavelength of $850 \mathrm{~nm}$ is 112 cycles/deg (2.66 $\mu \mathrm{m}$ diffraction-limit resolution), so the AO-fundus images in Figs. 10(b) and 10(c) were relatively close to the Raleigh diffraction limit, and the photoreceptor blurriness of AO-fundus images is not due to wavefront errors in the AO correction but rather due to the lack of resolution.

For this subject at the same location, the highest resolvable spatial frequencies in Figs. 10(d) and 10(e) were about 140 and 145 cycles/deg, which were close to or even better than the Raleigh diffraction limit of 141 cycles/deg $(2.10 \mu \mathrm{m}$ diffraction-limit resolution for the $6.62-\mathrm{mm}$ pupil size). It is obvious that the resolution of the AOSLO images is much higher than AO-fundus images under the same conditions. The AOSLO utilized a shorter laser wavelength for illumination than the commercial AO fundus, and the bimorph DM-based AO achieved an aberration compensation close to the diffraction limit. Owing to the improvement of resolution in this AOSLO prototype, many more details in retina diseases will be resolved.

Early signs of RP include loss of photoreceptors and changes in retinal vessels, especially thinning of small vessels and capillaries that are likely to develop in the inner retinal layers. 

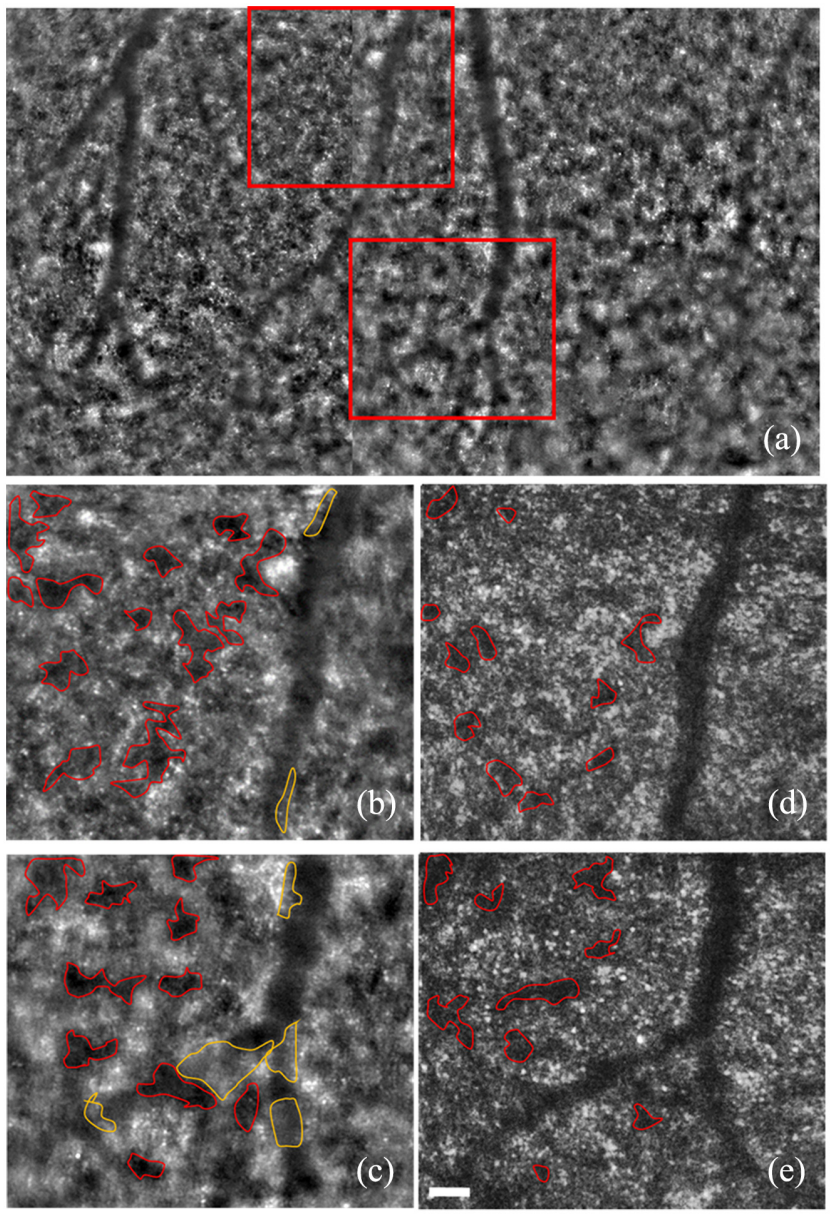

Fig. 10 High-resolution $\mathrm{AO}$ images of a female patient with $\mathrm{RP}$ at the age of 20. (a) Commercial AO-fundus image of photoreceptor cells with a FOV of $6 \mathrm{deg} \times 4 \mathrm{deg}(\mathrm{OD}$, temporal $=4 \mathrm{deg}$; superior $=3 \mathrm{deg})$. (b), (c) Enlarged regions of interest from the AO-fundus image indicated with the red squares in (a) (overlaid in red are the photoreceptor loss that were manually segmented by several experienced doctors). (d), (e) Single original images (1.7 deg $\times 1.5 \mathrm{deg}$ ) acquired with the AOSLO prototype at the same locations of (b) and (c), respectively (overlaid in red is the photoreceptor loss that was manually segmented by several experienced doctors). Scale bar is $50 \mu \mathrm{m}$.

Actually, conventional flood-illuminated imaging does not allow for axial resolution, even if AO is used in the AO-fundus camera RTX1 to correct aberrations, but the contrast is still reduced by light scattered from other retinal layers. Therefore, the AOSLO has the confocal pinhole for axial sectioning, which allows only light from near the plane of best focus to get imaged. Corresponding images of three retinal layers of this RP patient are shown in Fig. 12, and the sections reveal different retinal surfaces 1.8-deg superior to the fovea. Typical images of photoreceptor in Fig. 12(a) indicated that photoreceptors or pigments are concentrated in the front of the blood vessels, covering part of the blood vessels, or along the blood vessels, and most of them are found in the branches of the blood vessels (red-labeled ellipse areas). In contrast to an image where the focus is set to the photoreceptor layer, most vessels appear bright in Figs. 12(c) and 12(d) with the focus set to the superficial layers, because light from the highly scattering blood constituents and light reflection from the top vessel wall can now be collected by the confocal pinhole for imaging. In general, very small capillaries [size similar to the cone photoreceptors, which have been indicated by the red arrows in Figs. 12(c) and 12(d)] can be resolved with the axial section, and these characteristics can be observed to provide more information for retina pathology. In addition, it should be noted that RP causes a narrowing of retinal vessels (indicated by red lines) in inner layers of Fig. 12(c).

High-resolution and high-contrast imaging of several retinal layers can be achieved using this AOSLO, and small vessels can be resolved to provide more information for pathology. In practice, the added refractive power for axial sectioning were $-0.1 \mu \mathrm{m}$ (0.09D), $-0.2 \mu \mathrm{m}$ (0.25D), $-0.5 \mu \mathrm{m}$ (0.58D), and $-0.8 \mu \mathrm{m}$ (0.9D), respectively, for these images in Fig. 12 . The estimated thickness between the photoreceptor layer and the nerve fiber layer was $\sim 215 \mu \mathrm{m}$. In general, the axial sections of RP appear a little dark and show typically weak contrast (retinal images in Fig. 12 have been promoted in intensity), which is due to the weak scattering of light for AO imaging and can be greatly increased through an offset pinhole configuration ${ }^{48}$ or through a fluorescein angiography AOSLO setup in the future work.

\section{Discussion}

AO has been coupled with several different retinal imaging techniques to correct optical aberrations introduced by the cornea and lens of the eye and visualize fine retinal structures in living eyes. The combination of AO with scanning laser ophthalmoscopy (SLO) has been extensively used to generate impressive images of the human retina with unprecedented resolution, allowing for cellular-level imaging of human retina in vivo.

In this study, we developed an AOSLO system using a multiactuator bimorph DM with large strokes for human retina imaging in vivo. We also assembled a clinical prototype, which is a significant advancement in the field of AOSLO imaging since previous designs have only been feasible for laboratory use. In terms of the system design, a single bimorph DM was adopted to provide a compact and cost-effective solution for $\mathrm{AO}$ imaging of the diseased retina. In terms of AO implementation, the bimorph DM was operated with a successive AO wavefront control mode. In addition to its easy-to-use wavefront correction, the bimorph DM-based AO controller was capable of highly accurate aberration correction and was able to image almost every subject we tested. In this preliminary report, we demonstrate bimorph DM-based AO performance, SLO imaging of cone photoreceptors and blood vessels, and initial cell mosaics in a subject with RP.

Considering the routine ability of AOSLO to resolve photoreceptor cells, different AOSLO systems have been constructed to show cell mosaics throughout healthy and diseased retina, mostly distinguished by DM technology. ${ }^{25,27-30,38,39}$ Relative to our previous works, ${ }^{7,9,17}$ a low-cost bimorph DM with large stroke has been developed to realize an easy-to-use AOSLO platform, and a strategy for successive AO control of aberration correction is proposed. The large stroke of the bimorph DM permits the open-loop compensation for low-order aberrations with average RMS wavefront errors of approximately up to $4 \mu \mathrm{m}$, and then the closed-loop correction of high-order aberrations is validated through the convergence of membrane to acceptable RMS errors of $<0.08 \mu \mathrm{m}$ in all subjects once tested, ensuring AO performances close to the diffraction limit. The proposed AO successive control works with adequate precision for aberration correction and is implemented with SLO imaging in only one computer to make it easy to use. Facilitated with an HSWS, 

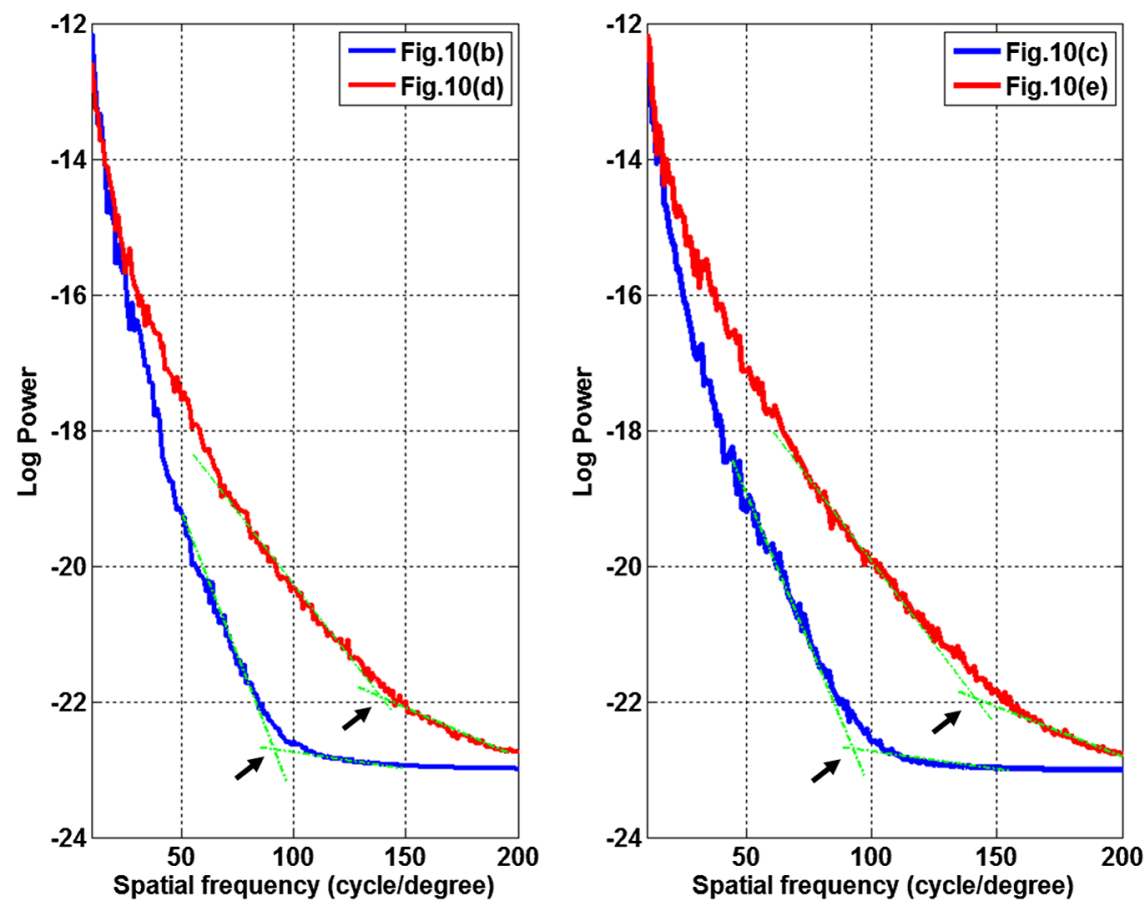

Fig. 11 The spectral power plot for retina images in Fig. 10. The green dot-dash lines were added to show the trends of the spectral power curves.
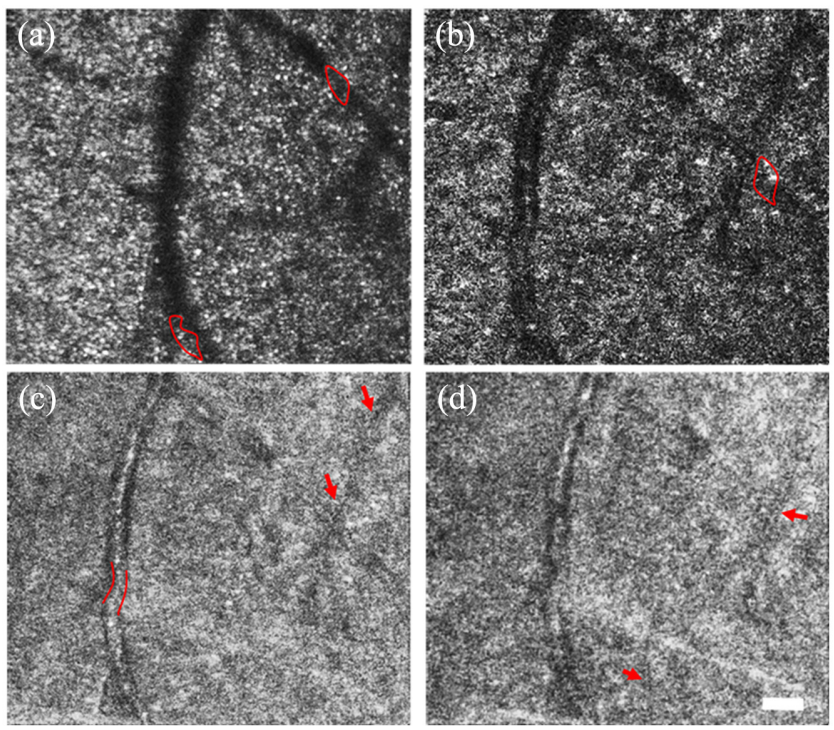

Fig. 12 Retinal images acquired at several retinal depths of the RP patient in Fig. 10. (a) Photoreceptor layer (overlaid in red-labeled ellipse are the photoreceptors or pigments concentrations that were manually segmented by several experienced doctors). (b) Between photoreceptor and blood vessel layers. (c) Blood vessel layer (overlaid in red arrows are small capillaries with size similar to the cone photoreceptors, and red lines are added to indicate a narrowing of retinal vessels cause by RP). (d) Nerve fiber layer (overlaid in red arrows are small capillaries with size similar to the cone photoreceptors). The scale bar is $50 \mu \mathrm{m}$.

the closed-loop AO compensation of ocular aberrations is accomplished at $\sim 10 \mathrm{~Hz}$ and is sufficient to compensate for the dynamic changes of eye aberrations. ${ }^{21}$

The other major issue is related to the optimization of compact optical design in a reflective AOSLO system. The design must avoid spurious reflections of lenses, which can be comparable to the light scattered back from the retina, even if the lenses are appropriately coated with an antireflection treatment. For practical purposes, off-axis spherical mirrors were used rather than lenses in this AOSLO system. However, the off-axis reflective implementation for scanning and imaging resulted in increased spherical aberrations and naturally introduced off-axis aberrations such as astigmatism and coma. To keep a finite conjugate setting of minimizing the off-axis aberrations, spherical mirrors with long focal lengths (traditionally more than $1000 \mathrm{~mm}$ ) can be employed to reduce off-axis angles, but this will increase the overall size of the system. In the current implementation, we folded spherical mirrors in a nonplanar configuration to minimize off-axis aberrations. By alternating the angle of the reflection of the beam of spherical mirrors M5 and M6 from the horizontal to vertical direction, the astigmatism aberrations were minimized. The compact optical design achieved a diffraction-limited performance for a vergence range of 10D (three different vergences for $-5 \mathrm{D}, 0 \mathrm{D}$, and $+5 \mathrm{D}$ ) over a $3 \mathrm{deg} \times 3$ deg FOV. Our AOSLO system has the capability of observing individual photoreceptor cells not only in healthy subjects but also in patients suffering from RP. AOSLO images of the diseased retina have much higher resolutions than those captured by the commercial AO-fundus camera, and AOSLO can produce much more accurate imaging of the photoreceptor mosaic.

Although this design allowed bimorph DM-based AOSLO to be implemented for a clinical prototype setting and successfully performed imaging for individual retina, the design has some limitations. For practical purposes, we developed a bimorph DM with an aperture of $20 \mathrm{~mm}$ to optimize the optical design of the AOSLO, which constrained the focal length of spherical mirrors. In addition, the requirement of pupil diameter larger than $\sim 6 \mathrm{~mm}$ might not always be possible in some patients. In one of our previous experiments, an elder subject had a usable 
pupil diameter of 4 to $5 \mathrm{~mm}$ because of age-related miosis and failed to be imaged with AO correction of ocular aberrations. Actually, the AOSLO imaging is very likely to fail, once the shape of pupil is abnormal (affected by injury or innate); in such situation, the wavefront cannot be measured accurately over the entire pupil. It would be useful to minimize these problems as much as possible. Therefore, in future studies, we plan to develop a bimorph DM with higher strokes of up to $\sim 100 \mu \mathrm{m}$ and smaller aperture size of $\sim 10 \mathrm{~mm}$, allowing a smaller pupil of $\sim 4 \mathrm{~mm}$ to be used to image for a wider clinical applicability of this instrument. In addition, an HSWS will be designed to achieve more accurate wavefront measurements, which is particularly good for abnormal pupils or artificial lens that is inserted after cataract surgery. We also plan to produce a much more compact system design where more mirrors will not be confined to a single plane.

It is clear that almost all AOSLO systems achieve very good imaging of both healthy and diseased human retina, benefitting from the AO correction of aberrations. However, it is rather hard to measure the improvements in AOSLO imaging. In an imaging system, the image resolution is governed by the optical modulation transfer function, and typically the Rayleigh criterion is preferred for an estimate of resolution. ${ }^{49}$ In a conventional microscope with a high Strehl ratio and low noise, it is reasonable to expect it to be able to perform better than the Rayleigh criterion. In the AOSLO system, the real resolution still depends on the signal-to-noise ratio, although the confocal diffraction limit can reach $r_{\text {confocal }}=0.88 \lambda f / D$, which is approximately $1.4 \times$ better than the Rayleigh criterion, by using an ideal confocal pinhole. However, the confocal resolution requires a very small confocal pinhole conjugate to the retina by collecting light backscattered only from the plane of focus while minimizing light multiply scattered from out of the plane of focus. In Table 1, we compared the resolution performances under different conditions, and we can conclude that the estimated resolutions in most subjects were able to achieve close to or even better than the Rayleigh criterion. To obtain higher signal intensity and image brightness, the pinhole size we used was $50 \mu \mathrm{m}$, which was $\sim 1.44$ times the size of the Airy disk diameter; this resulted in better signal throughput while sacrificing some resolution.
Therefore, the current AOSLO system did not achieve the confocal diffraction-limit resolution due to many constraints. The most important issue to be addressed in future work is that the AO correction still needs to be improved to reach the confocal diffraction-limited retinal imaging with a Strehl ratio value of $\lambda / 21$.

In the current study, we compared the retinal imaging between our AOSLO system and the commercial AO-fundus camera. Although this comparison is somewhat arbitrary and unreasonable, the AO-fundus camera was selected for the comparison because it was the only imaging system available on the market that contained a DM with dozens of strokes and that could be used for retinal imaging including RP diseased retinal imaging. It was hypothesized that the confocal imaging would produce a much higher resolution than the flood illuminationbased fundus camera. As expected, the loss of photoreceptor mosaic could be distinguished more accurately with the AOSLO prototype than with the commercial AO-fundus camera, which indicates that the bimorph DM achieved a more accurate $\mathrm{AO}$ correction and higher resolution improvement. One advantage of the commercial AO-fundus camera is its flexibility and convenience for clinical operations, so our future studies will aim to develop automated image acquisition and analysis techniques to further address the difficulties that arise from system complexity.

In the optical design of AOSLO, it is desirable to enable acquisitions of retinal imaging in an FOV of $3 \mathrm{deg} \times 3 \mathrm{deg}$. One disadvantage of our current AOSLO system is caused by the physical limits of the horizontal scanner with a 5-deg peak-to-peak value for optical angles, thereby producing a maximum horizontal angle of $2 \mathrm{deg}$ on the retina. We, therefore, limited the real imaging FOV to $1.7 \mathrm{deg} \times 1.5 \mathrm{deg}$ considering the influence of duty cycle and nonlinear resonance scanning mode. However, it would be useful to maximize the FOV as much as possible in future designs by using horizontal scanners, such as MEMS scanners with larger scanning angles while retaining high-frequency scanning.

Although the clinical prototype for this bimorph DM-based AOSLO is completed, many more objectives must be completed in the next research phase. This AOSLO platform will be widely

Table 1 Resolution comparisons between Rayleigh criteria, confocal diffraction-limit, and estimated resolution with human eyes.

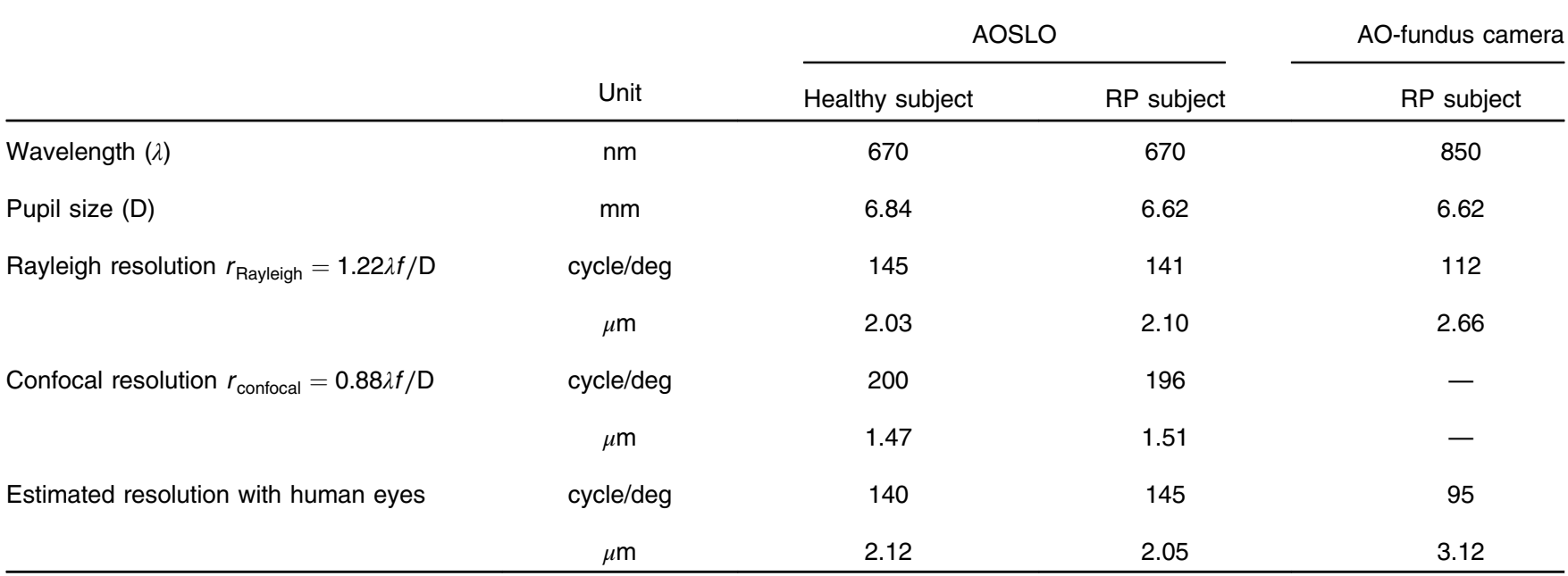

Note: In the current AOSLO imaging, human eye has a focal length of $17 \mathrm{~mm}$, and the confocal pinhole size is $\Phi 50 \mu \mathrm{m}$. Note that the healthy and RP subjects are the same as before in this article. 
validated in an ophthalmology clinic to provide qualitative and quantitative information on photoreceptor-related retinal diseases, including age-related macular degeneration, macular telangiectasia, and RP. Not only the photoreceptors but also other fine-scale structures such as vasculature, retinal pigment epithelium, and ganglion cells are crucial to the health and functioning of the retina and are particularly important for understanding and treating many retinal diseases. Since the correction of ocular aberrations with AO is essential to resolve photoreceptor and vessels images, the capabilities of the next AOSLO system will be expanded to image more retinal structures, resulting in a multifunctional piece of equipment suitable for researching and diagnosing multiple retinal diseases.

\section{Conclusion}

Significant progress was made in the system design of the bimorph DM-based AOSLO system to develop a clinical retina imaging prototype. A low-cost bimorph DM with a large stroke of $50 \mu \mathrm{m}$ and an aperture of $20 \mathrm{~mm}$ was utilized to realize a strategy for successive AO control of aberration correction for the first time. This control strategy permitted open-loop compensation for low-order aberrations with average RMS wavefront errors of approximately up to $4 \mu \mathrm{m}$ and closed-loop correction of high-order aberrations to acceptable RMS errors of $<0.08 \mu \mathrm{m}$ in all subjects once tested. Spherical mirrors were folded in a nonplanar configuration to minimize off-axis aberrations and provide a compact, cost-effective design, which achieved a diffraction-limited performance for a vergence range of 10D (three different vergences for $-5 \mathrm{D}, 0 \mathrm{D}$, and $+5 \mathrm{D}$ ) over a 3 deg $\times 3$ deg FOV. We built and tested this AOSLO platform capable of observing individual photoreceptor cells and blood vessels not only in healthy subjects but also in patients suffering from RP. AOSLO images of the diseased retina had much higher resolutions than those captured by the commercial AO-fundus camera. Owing to the improvement of resolution, photoreceptor mosaic losses were distinguished more accurately with this prototype than with the commercial AO-fundus camera. The compact design and easy handling of bimorph DM-based AO control may facilitate the quick translation of AOSLO into clinical settings, and this prototype development will continue with future device refinement and extensive clinical testing.

\section{Disclosures}

No conflicts of interest, financial or otherwise, are declared by the authors.

\section{Acknowledgments}

This work is supported by the Jiangsu Province Key R\&D Program (Grant Nos. BE2019682, BE2018667), the National Natural Science Foundation of China (Grant Nos. 61605210, 61675226, and 61378090), the National Key R\&D Program of China (Grant Nos. 2016YFC0102500, 2017YFB0403700), the Pilot Project of the Chinese Academy of Sciences (Grant No. XDB02060000), the Frontier Science research project of the Chinese Academy of Sciences (Grant No. QYZDB-SSWJSC03); the Strategic Priority Research Program of the Chinese Academy of Sciences (Grant No. XDB02060000), Youth Innovation Promotion Association of Chinese Academy of Sciences (Grant No. 2019320), the National Instrumentation Program (Grant No. 2012YQ120080), and the Zhejiang Province Technology Program (Grant No. 2013C33170). We also thank LetPub (www.letpub.com) for linguistic assistance during the preparation of this article.

\section{References}

1. A. Roorda et al., "Adaptive optics scanning laser ophthalmoscopy," Opt. Express 10, 405-412 (2002).

2. D. R. Williams, "Imaging single cells in the living retina," Vision Res. 51, 1379-1396 (2011).

3. H. Song et al., "Cone and rod loss in Stargardt disease revealed by adaptive optics scanning light ophthalmoscopy," JAMA Ophthalmol. 133(10), 1198-1203 (2015).

4. J. Tam and A. Roorda, "Speed quantification and tracking of moving objects in adaptive optics scanning laser ophthalmoscopy," J. Biomed. Opt. 16(3), 036002 (2011).

5. E. Rossi and A. Roorda, "The relationship between visual resolution and cone spacing in the human fovea," Nat. Neurosci. 13, 156-157 (2010).

6. T. Yamaguchi et al., "Adaptive optics dioptric scanning ophthalmoscope with a wider field of view similar to those of normal ophthalmoscopes," Opt. Lett. 37, 2496-2498 (2012).

7. M. Land et al., "Cone structure in subjects with known genetic relative risk for AMD," Optom. Vision Sci. 91(8), 939-949 (2014).

8. T. J. Zhang et al., "Variability in human cone topography assessed by adaptive optics scanning laser ophthalmoscopy," Am. J. Ophthalmol. 160(2), 290-300.e1 (2015).

9. M. Georgiou et al., "Adaptive optics imaging of inherited retinal diseases," Br. J. Ophthalmol. 102(8), 1028-1035 (2018).

10. D. Cunefare et al., "Automatic detection of cone photoreceptors in split detector adaptive optics scanning light ophthalmoscope images," Biomed. Opt. Express 7, 2036-2050 (2016).

11. S. A. Burns et al., "Adaptive optics imaging of the human retina," Prog. Retinal Eye Res. 68, 1-30 (2019).

12. F. Vargas-Martin, P. M. Prieto, and P. Artal, "Correction of the aberrations in the human eye with a liquid crystal spatial light modulator: limits to performance," J. Opt. Soc. Am. A 15, 2552-2562 (1998).

13. T. Shirai, "Liquid-crystal adaptive optics based on feedback interferometry for high-resolution retinal imaging," Appl. Opt. 41, 4013-4023 (2002).

14. E. Dalimier and C. Dainty, "Comparative analysis of deformable mirrors for ocular adaptive optics," Opt. Express 13, 4275-4285 (2005).

15. N. Doble and D. R. Williams, "The application of MEMS technology for adaptive optics in vision science," IEEE J. Sel. Top. Quantum Electron. 10, 629-635 (2004).

16. K. Morzinski et al., "Stroke saturation on a MEMS deformable mirror for woofer-tweeter adaptive optics," Opt. Express 17(7), 5829-5844 (2009).

17. Z. Zhong et al., "In vivo measurement of erythrocyte velocity and retinal blood flow using adaptive optics scanning laser ophthalmoscopy," Opt. Express 16, 12746-12756 (2008).

18. H. Li et al., "Measurement of oxygen saturation in small retinal vessels with adaptive optics confocal scanning laser ophthalmoscope," $J$. Biomed. Opt. 16(11), 110504 (2011).

19. H. Li et al., "Automatic montage of retinal images in adaptive optics confocal scanning laser ophthalmoscope," Opt. Eng. 51(5), 057008 (2012).

20. J. Liang and D. R. Williams, "Aberrations and retinal image quality of the normal human eye," J. Opt. Soc. Am. A 14, 2873-2883 (1997).

21. J. Porter, A. Guirao, and D. R. Williams, "Monochromatic aberrations of the human eye in a large population," J. Opt. Soc. Am. A 18, 1793-1803 (2001).

22. J. Lu et al., "Adaptive optics parallel near-confocal scanning ophthalmoscopy," Opt. Lett. 41, 3852-3855 (2016).

23. J. Lu et al., "High speed adaptive optics ophthalmoscopy with an anamorphic point spread function," Opt. Express 26, 14356-14374 (2018).

24. B. Gu et al., "Noninvasive in vivo characterization of erythrocyte motion in human retinal capillaries using high-speed adaptive optics nearconfocal imaging," Biomed. Opt. Express 9, 3653-3677 (2018).

25. C. Li et al., "A correction algorithm to simultaneously control dual deformable mirrors in a woofer-tweeter adaptive optics system," Opt. Express 18(16), 16671-16684 (2010). 
26. J. Zhang et al., "An adaptive optics imaging system designed for clinical use," Biomed. Opt. Express 6(6), 2120-2137 (2015).

27. A. Roorda and J. L. Duncan, "Adaptive optics ophthalmoscopy," Annu. Rev. Vision Sci. 1, 19-50 (2015).

28. D. Merino and P. Loza-Alvarez, "Adaptive optics scanning laser ophthalmoscope imaging: technology update," Clin. Ophthalmol. 10, 743-755 (2016).

29. S. Marcos et al., "Vision science and adaptive optics, the state of the field," Vision Res. 132, 3-33 (2017).

30. B. Zhang et al., "Adaptive optics scanning laser ophthalmoscopy in fundus imaging, a review and update," Int. J. Ophthalmol. 10(11), 1751-1758 (2017).

31. Y. He et al., "Design of a compact, bimorph deformable mirror-based adaptive optics scanning laser ophthalmoscope," in Oxygen Transport to Tissue XXXIII, Q. Luo et al., Eds., Advances in Experimental Medicine and Biology, Vol. 923, pp. 375-383, Springer, Cham (2016).

32. Y. Wang et al., "Bimorph deformable mirror based adaptive optics scanning laser ophthalmoscope for retina imaging in vivo," Chin. Opt. Lett. 15, 121102 (2017).

33. A. H. Tunnacliffe, Introduction to Visual Optics, Association of British Dispensing Opticians, England (1989).

34. Y. Zhang, S. Poonja, and A. Roorda, "MEMS-based adaptive optics scanning laser ophthalmoscopy," Opt. Lett. 31, 1268-1270 (2006).

35. J. Lu et al., "Superresolution in adaptive optics confocal scanning laser ophthalmoscope," Acta Phys. Sin. 60(3), 034207 (2011).

36. H. Li et al., "Tracking features in retinal images of adaptive optics confocal scanning laser ophthalmoscope using KLT-SIFT algorithm," Biomed. Opt. Express 1, 31-40 (2010).

37. S. A. Burns et al., "Large field of view, modular, stabilized, adaptive optics-based scanning laser ophthalmoscope," J. Opt. Soc. Am. A 24, 1313-1326 (2007).

38. J. Lu et al., "Retina imaging in vivo with the adaptive optics confocal scanning laser ophthalmoscope," Proc. SPIE 7519, 75191I (2009).

39. W. Zou, X. Qi, and S. A. Burns, "Woofer-tweeter adaptive optics scanning laser ophthalmoscopic imaging based on Lagrange-multiplier damped least-squares algorithm," Biomed. Opt. Express 2, 1986-2004 (2011).

40. S. A. Burns et al., "Robust adaptive optics systems for vision science," Proc. SPIE 10502, 1050209 (2018).

41. F. Felberer et al., "Lens based adaptive optics scanning laser ophthalmoscope," Opt. Express 20(16), 17297-17310 (2012).

42. American National Standard Institute, "American National Standard for safe use of lasers," ANSI Z 136-1 (2000).

43. L. N. Thibos et al., "Standards for reporting the optical aberrations of eyes," J. Refract. Surg. 18(5), S652-S660 (2002).

44. J. Zhao et al., "Statistical analysis of ocular monochromatic aberrations in Chinese population for adaptive optics ophthalmoscope design," J. Innovative Opt. Health Sci. 10, 1650038 (2017).

45. J. I. Yellott et al., "Spectral analysis of spatial sampling by photoreceptors: topological disorder prevents aliasing," Vision Res. 22(9), 1205-1210 (1982).

46. D. Hartong, E. Berson, and T. Dryja, "Retinitis pigmentosa," The Lancet 368(9549), 1795-1809 (2006).
47. "Facts about retinitis pigmentosa," National Eye Institute (2014).

48. S. A. Burns et al., "In vivo adaptive optics microvascular imaging in diabetic patients without clinically severe diabetic retinopathy," Biomed. Opt. Express 5(3), 961-974 (2014).

49. G. Boreman, Modulation Transfer Function in Optical and ElectroOptical Systems, SPIE Press, Bellingham, Washington, Eq. (1.28) (2001).

Yuanyuan Wang received her $\mathrm{PhD}$ in optics from Chinese Academy of Sciences in 2018. Currently, she is an assistant professor at the School of Ophthalmology and Optometry and Eye Hospital. Her main research interest is in retina imaging.

Yi He is an assistant professor at the Jiangsu Key Laboratory of Medical Optics, Suzhou Institute of Biomedical Engineering and Technology, Chinese Academy of Sciences. He received his BS degree in automation from the University of Science and Technology of China in 2008, and his MS degree and PhD from Chinese Academy of Sciences in 2013. His current research interests include optical engineering, biomedical imaging, and optoelectronic systems.

Ling Wei received his MS degree and $\mathrm{PhD}$ in optics from Chinese Academy of Sciences in 2012. Currently, he is an assistant professor at Institute of Optics and Electronics Chinese Academy of Sciences. His main research interest is in imaging processing and adaptive optics (AO).

Jinsheng Yang received his $\mathrm{PhD}$ in optics from Chinese Academy of Sciences in 2009. Currently, he is an assistant professor at Institute of Optics and Electronics Chinese Academy of Sciences. His main research interest is in optical design and $\mathrm{AO}$.

Xiqi Li received his $\mathrm{PhD}$ in optics from Chinese Academy of Sciences in 2013. Currently, he is an assistant professor at Institute of Optics and Electronics Chinese Academy of Sciences. His main research interest is in biomedical imaging and image processing.

Hong Zhou received his $\mathrm{PhD}$ in optics from Chinese Academy of Sciences in 2013. Currently, he is an assistant professor at Institute of Optics and Electronics Chinese Academy of Sciences. His main research interest is in $\mathrm{AO}$.

Guohua Shi received his MS degree and $\mathrm{PhD}$ in optics from Chinese Academy of Sciences in 2011. Currently, he is a professor at the Jiangsu Key Laboratory of Medical Optics, Suzhou Institute of Biomedical Engineering and Technology, Chinese Academy of Sciences. His main research interest is in $\mathrm{AO}$ and biomedical imaging.

Yudong Zhang received his MS degree and PhD in optics from Chinese Academy of Sciences in 1987 and 1991, respectively. Currently, he is a research professor at Institute of Optics and Electronics Chinese Academy of Sciences. His main research interest is in $\mathrm{AO}$. 